\title{
EVIDENCE FOR CO SHOCK EXCITATION IN NGC 6240 FROM HERSCHEL SPIRE SPECTROSCOPY
}

\author{
R. Meijerink ${ }^{1,2}$, L. E. Kristensen ${ }^{2}$, A. Wei ${ }^{3}$, P. P. van der $\mathrm{WerF}^{2}$, F. Walter ${ }^{4}$, M. SpaAns ${ }^{1}$, A. F. Loenen ${ }^{2}$, J. Fischer ${ }^{5}$,
}

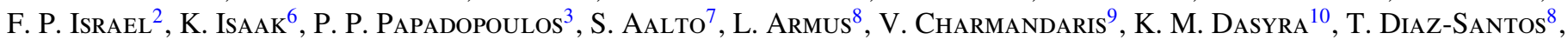
A. Evans ${ }^{11,12}$, Y. GaO ${ }^{13}$, E. GonZÁlez-Alfonso ${ }^{14}$, R. GÜSTEN ${ }^{3}$, C. HenKel $^{3}{ }^{15}$, C. Kramer ${ }^{16}$, S. Lord ${ }^{17}$, J. MarTín-Pintado ${ }^{18}$,

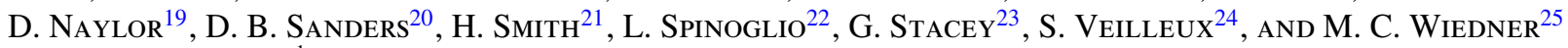

${ }^{1}$ Kapteyn Astronomical Institute, P.O. Box 800, NL-9700 AV Groningen, The Netherlands

${ }^{2}$ Leiden Observatory, Leiden University, P.O. Box 9513, NL-2300 RA Leiden, Netherlands

${ }^{3}$ Max-Planck-Institut für Radioastronomie, Auf dem Hügel 16, Bonn, D-53121, Germany

${ }^{4}$ Max-Planck-Institut für Astronomie, Königstuhl 17, Heidelberg, D-69117, Germany

${ }^{5}$ Naval Research Laboratory, Remote Sensing Division, Washington, DC 20375, USA

${ }^{6}$ ESA Astrophysics Missions Division, ESTEC, P.O. Box 299, NL-2200 AG Noordwijk, The Netherlands

${ }^{7}$ Department of Radio and Space Science, Onsala Observatory, Chalmers University of Technology, SE-43992 Onsala, Sweden

${ }^{8}$ Spitzer Science Center, California Institute of Technology, MS 220-6, Pasadena, CA 91125, USA

${ }^{9}$ University of Crete, Department of Physics, GR-71003 Heraklion, Greece

${ }^{10}$ Observatoire de Paris, LERMA (CNRS:UMR8112), 61 Av. de l'Observatoire, F-75014, Paris, France

${ }^{11}$ Department of Astronomy, University of Virginia, 530 McCormick Road, Charlottesville, VA 22904, USA

${ }_{12}$ National Radio Astronomy Observatory, 520 Edgemont Road, Charlottesville, VA 22903, USA

${ }^{13}$ Purple Mountain Observatory, Chinese Academy of Sciences, 2 West Beijing Road, Nanjing 210008, China

${ }^{14}$ Universidad de Alcalá Henares, Departamente de Física, Campus Universitario, E-28871 Alcalá de Henares, Madrid, Spain

${ }^{15}$ Astron. Dept., King Abdulaziz University, P.O. Box 80203, Jeddah, Saudi Arabia

${ }^{16}$ Instituto Radioastronomie Millimetrica (IRAM), Av. Divina Pastora 7, Nucleo Central, E-18012 Granada, Spain

${ }^{17}$ NASA Herschel Science Center, California Institute of Technology, M.S. 10022, Pasadena, CA 91125, USA

${ }^{18}$ Departamento de Astrofisica Molecular e Infrarroja-Instituto de Estructura de la Materia-CSIC, Calle Serrano 121, E-28006 Madrid, Spain

${ }^{19}$ Institute for Space Imaging Science, Department of Physics and Astronomy, University of Lethbridge, Lethbridge, Alberta, T1K 3M4, Canada

${ }^{20}$ University of Hawaii, Institute for Astronomy, 2680 Woodlawn Drive, Honolulu, HI 96822, USA

${ }^{21}$ Harvard-Smithsonian Center for Astrophysics, 60 Garden Street, Cambridge, MA 02138, USA

${ }^{22}$ Istituto di Astrofisica e Planetologia Spaziali, INAF, Via Fosso del Cavaliere 100, I-00133, Roma, Italy

${ }^{23}$ Department of Astronomy, Cornell University, Ithaca, NY 14853, USA

${ }^{24}$ Department of Astronomy, University of Maryland, College Park, MD 20742, USA

${ }^{25}$ Observatoire de Paris, LERMA, CNRS, 61 Av. de l'Observatoire, F-75014 Paris, France

Received 2012 July 27; accepted 2012 November 28; published 2012 December 13

\begin{abstract}
We present Herschel SPIRE FTS spectroscopy of the nearby luminous infrared galaxy NGC 6240. In total 20 lines are detected, including $\mathrm{CO} J=4-3$ through $J=13-12,6 \mathrm{H}_{2} \mathrm{O}$ rotational lines, and [ $\mathrm{C} \mathrm{I}$ ] and [ $\mathrm{N}$ II] fine-structure lines. The CO to continuum luminosity ratio is 10 times higher in NGC 6240 than Mrk 231. Although the CO ladders of NGC 6240 and Mrk 231 are very similar, UV and/or X-ray irradiation are unlikely to be responsible for the excitation of the gas in NGC 6240. We applied both $\mathrm{C}$ and $\mathrm{J}$ shock models to the $\mathrm{H}_{2} v=1-0 S(1)$ and $v=2-1 S(1)$ lines and the $\mathrm{CO}$ rotational ladder. The CO ladder is best reproduced by a model with shock velocity $v_{s}=10 \mathrm{~km} \mathrm{~s}^{-1}$ and a pre-shock density $n_{\mathrm{H}}=5 \times 10^{4} \mathrm{~cm}^{-3}$. We find that the solution best fitting the $\mathrm{H}_{2}$ lines is degenerate. The shock velocities and number densities range between $v_{s}=17-47 \mathrm{~km} \mathrm{~s}^{-1}$ and $n_{\mathrm{H}}=10^{7}-5 \times 10^{4} \mathrm{~cm}^{-3}$, respectively. The $\mathrm{H}_{2}$ lines thus need a much more powerful shock than the $\mathrm{CO}$ lines. We deduce that most of the gas is currently moderately stirred up by slow $\left(10 \mathrm{~km} \mathrm{~s}^{-1}\right)$ shocks while only a small fraction $(\lesssim 1 \%)$ of the interstellar medium is exposed to the high-velocity shocks. This implies that the gas is rapidly losing its highly turbulent motions. We argue that a high CO line-to-continuum ratio is a key diagnostic for the presence of shocks.
\end{abstract}

Key words: galaxies: active - galaxies: individual (NGC 6240) - galaxies: nuclei - galaxies: starburst infrared: galaxies

\section{INTRODUCTION}

We present Herschel $^{26}$ SPIRE FTS (Griffin et al. 2010) observations of the nearby luminous infrared galaxy NGC 6240 (IRAS 16504+0228, UGC 10592). With a redshift of $z=$ 0.024576 and a 7 year Wilkinson Microwave Anisotropy Probe flat cosmology $\left(H_{0}=70.50 \mathrm{~km} \mathrm{~s}^{-1} \mathrm{Mpc}^{-1}, \Omega_{\text {matter }}=0.27\right.$, $\Omega_{\text {vacuum }}=0.73$ ) NGC 6240 is at a luminosity distance of $D_{\mathrm{L}}=107 \mathrm{Mpc}$, with $1^{\prime \prime}$ corresponding to $492 \mathrm{pc}$. The derived $8-1000 \mu \mathrm{m}$ luminosity of the merger galaxy NGC 6240 is $L_{\mathrm{IR}}=7.5 \times 10^{11} L_{\odot}$. Different power sources are suggested for this infrared luminosity, which complicate the interpretation

${ }^{26}$ Herschel is an ESA space observatory with science instruments provided by European-led Principal Investigator consortia and with important participation from NASA. of observations. The source is a strong X-ray emitter. Based on modeling of Chandra between 0.5 and $8 \mathrm{keV}$, Komossa et al. (2003) derive extinction corrected luminosities of $L_{\mathrm{X}, N}=$ $1.9 \times 10^{42} \mathrm{erg} \mathrm{s}^{-1}$ and $L_{\mathrm{X}, S}=0.7 \times 10^{42} \mathrm{erg} \mathrm{s}^{-1}$ for the northern and southern active galactic nucleus cores in the energy range 0.1-10 keV. However, using BeppoSAX observations, Vignati et al. (1999) find that the intrinsic hard X-ray luminosity shows up above $9-10 \mathrm{keV}$, and their models of the higher energy yields $L(2-10 \mathrm{keV}) \approx 3.6 \times 10^{44} \mathrm{erg} \mathrm{s}^{-1}$. Hydrogen recombination lines (Rieke et al. 1985; Depoy et al. 1986; Elston \& Maloney 1990; van der Werf et al. 1993) and luminous polycyclic aromatic hydrocarbon (PAH) emission (Armus et al. 2006) in the two nuclei indicate recent star formation. The $\mathrm{H}_{2} v=1-0 S(1)$ $2.12 \mu \mathrm{m}$ line, presented by van der Werf et al. (1993), shows a peak in the overlap region between these two nuclei, which are 


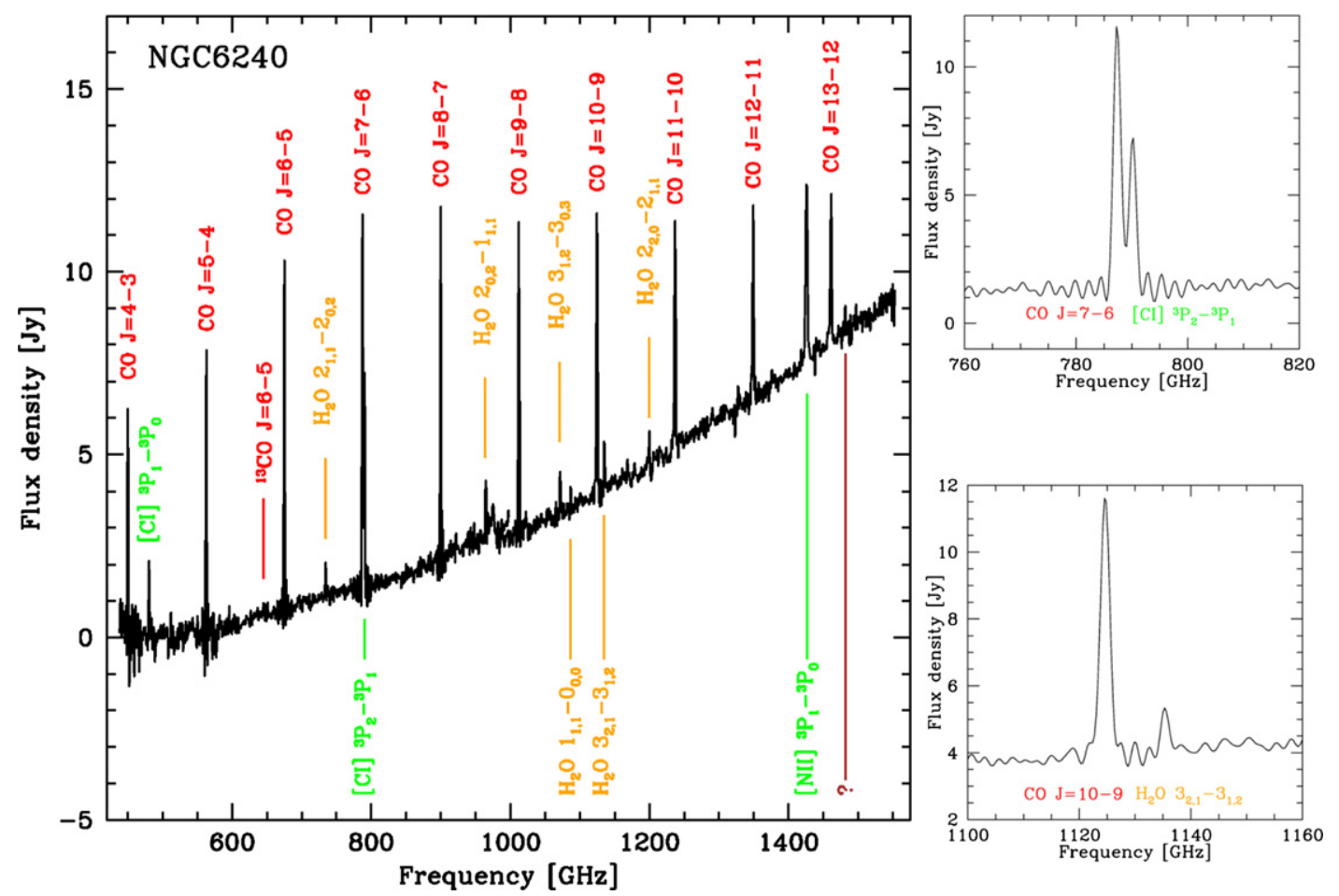

Figure 1. Full SPIRE FTS spectrum of NGC 6240 and zoom-in on line blends, with the frequency on the $x$-axis and the flux density in Jy on the $y$-axis. The detected lines are marked: $\mathrm{CO}$ (red), [C I] and [N $\mathrm{II}]$ (green), $\mathrm{H}_{2} \mathrm{O}$ (orange), and unidentified (brown). In the overlap region between the two frequency bands (958-989 $\mathrm{GHz}$ ), the noisy parts were clipped and plotted on top of each other.

$2^{\prime \prime}$ apart. This $\mathrm{H}_{2}$ emission extends over several kpc and shows a complex morphology. The authors conclude that the bright $\mathrm{H}_{2}$ emission between the nuclei is generated in shocks resulting from the collision of the interstellar media (ISM) of the merging galaxies. More recently, Engel et al. (2010) observed the same line at high spatial $(0,5)$ and spectral $\left(\sim 90 \mathrm{~km} \mathrm{~s}^{-1}\right)$ resolution. The line profiles also indicate multiple components, and the dispersion map suggests that the gas is highly disturbed and turbulent. Interferometric observations of several $\mathrm{CO}, \mathrm{HCN}$, and $\mathrm{HCO}^{+}$lines show a high-density peak between the two nuclei (Tacconi et al. 1999; Nakanishi et al. 2005; Iono et al. 2007). Tacconi et al. (1999) conclude that NGC 6240 is in an earlier merging stage than Arp 220, the prototypical ultra-luminous infrared galaxy, and state that the gas is in the process of settling between the two nuclei, and dissipating angular momentum rapidly. Engel et al. (2010) also presented a ${ }^{12} \mathrm{CO} J=2-1$ interferometric map, and conclude that the $\mathrm{H}_{2}$ and $\mathrm{CO}$ emission are coextensive, but do not coincide with the stellar emission distribution.

This Letter is ordered as follows. In Section 2, the observations, data reduction and line luminosities are discussed. In Section 3, we discuss the different excitation components (PDR, XDR, shocks) in combination with the geometry of NGC 6240. Consequently, we compose a CO ladder using both the available SPIRE FTS and ground-based CO data from Papadopoulos et al. (2012b) and compare this system to the ULIRG Mrk 231 (previously studied by us with Herschel; van der Werf et al. 2010). Then we analyze this with shock models from Kristensen et al. (2007). In Section 4, we conclude with a discussion and a summary of the results.

\section{OBSERVATIONS, DATA REDUCTION, AND RESULTS}

NGC 6240 was observed (Observations ID 1342214831) in staring mode with the SPIRE FTS on 2011 February 27, as part of the Herschel Open Time Key Program HerCULES (PI Van der Werf). The high spectral resolution mode was used, yielding a resolution of $1.2 \mathrm{GHz}$ over both observing bands: the low frequency band covering $\nu=447-989 \mathrm{GHz}(\lambda=671-303 \mu \mathrm{m})$ and the high frequency band covering $v=958-1545 \mathrm{GHz}$ $(\lambda=313-194 \mu \mathrm{m})$. In total 97 repetitions (194 FTS scans) were carried out, yielding an on source integration time of $12920 \mathrm{~s}(3.6 \mathrm{hr})$ for each band. A reference measurement was used to subtract the combined emission from the sky, telescope and instrument. The data were processed and calibrated using HIPE version 6.0. The extent of the CO $J=3-2$ emission is $4^{\prime \prime}$ (Wilson et al. 2008), while the SPIRE beam varies from $17^{\prime \prime}$ to $42^{\prime \prime}$ over our spectrum. Therefore, a point source calibration procedure was adopted, and no corrections for wavelength dependent beam coupling factors were necessary.

The full SPIRE FTS spectrum of NGC 6240 is shown in Figure 1. In the overlap region between the two frequency bands (958-989 GHz), the noisy parts of the two spectrometer bands were clipped and plotted on top of each other. A total of 20 lines were detected of which one line at the observed frequency $v_{\text {rest }}=1481.6 \mathrm{GHz}$ remains unidentified. We also see hints of absorption and emission between, e.g., 1300 and $1350 \mathrm{GHz}$. The significance of these features are still under investigation, and requires a more advanced flux extraction than currently adopted. The identified lines include the CO $J=4-3$ to $J=13-12,6 \mathrm{H}_{2} \mathrm{O}$ lines, [C I] 370 and $609 \mu \mathrm{m}$, and [N II] $205 \mu \mathrm{m}$. A well-constrained upper limit was obtained for the ${ }^{13} \mathrm{CO} J=6-5$ line. The line fluxes are listed in Table 1. In all cases a simple integration over the line was done. This procedure was repeated with approximately 100 different choices of baseline and integration intervals. Specifically, the integration is done from \pm 5 to $\pm 9 \mathrm{GHz}$ centered on the line with steps of $0.4 \mathrm{GHz}$ (10 integration borders). For each of these integration borders, the baseline window is set from \pm 2 to $\pm 3.5 \mathrm{GHz}$ with steps of $0.15 \mathrm{GHz}$ (10 iterations for each 
Table 1

Derived Fluxes from the SPIRE FTS Observations Supplemented with Ground-based Observations

\begin{tabular}{|c|c|c|c|c|}
\hline Line & $\begin{array}{c}v_{\mathrm{obs}} \\
(\mathrm{GHz})\end{array}$ & $\begin{array}{l}\lambda_{\text {rest }} \\
(\mu \mathrm{m})\end{array}$ & $\begin{array}{c}S_{\text {line }} \\
\left(\mathrm{Jy} \mathrm{km} \mathrm{s}^{-1}\right)\end{array}$ & $\begin{array}{l}L_{\text {line }} \\
\left(L_{\odot}\right)\end{array}$ \\
\hline $\mathrm{CO} J=1-0^{\mathrm{a}}$ & & 2600.76 & $322 \pm 29$ & $4.3 \times 10^{5, d}$ \\
\hline $\operatorname{CO} J=2-1^{\mathrm{a}}$ & & 1300.40 & $1490 \pm 250$ & $4.0 \times 10^{6}$ \\
\hline $\operatorname{CO} J=3-2^{\mathrm{a}}$ & & 866.963 & $3210 \pm 640$ & $1.2 \times 10^{7}$ \\
\hline $\operatorname{CO} J=4-3$ & 449.99 & 650.252 & $4630 \pm 370$ & $2.8 \times 10^{7}$ \\
\hline $\operatorname{CO} J=5-4$ & 562.41 & 520.231 & $5640 \pm 150$ & $3.8 \times 10^{7}$ \\
\hline $\operatorname{CO} J=6-5$ & 674.83 & 433.556 & $5910 \pm 82$ & $4.8 \times 10^{7}$ \\
\hline $\operatorname{CO~} J=7-6$ & 787.25 & 371.650 & $6010 \pm 60$ & $5.6 \times 10^{7}$ \\
\hline $\mathrm{CO} J=8-7$ & 899.68 & 325.225 & $5830 \pm 89$ & $6.3 \times 10^{7}$ \\
\hline $\mathrm{CO} J=9-8$ & 1012.1 & 289.120 & $4770 \pm 82$ & $5.7 \times 10^{7}$ \\
\hline $\operatorname{CO} J=10-9$ & 1124.5 & 260.240 & $4160 \pm 67$ & $5.6 \times 10^{7}$ \\
\hline $\mathrm{CO} J=11-10$ & 1236.9 & 236.613 & $3160 \pm 75$ & $4.7 \times 10^{7}$ \\
\hline $\mathrm{CO} J=12-11$ & 1349.1 & 216.927 & $2590 \pm 60$ & $4.2 \times 10^{7}$ \\
\hline $\operatorname{CO} J=13-12$ & 1461.2 & 200.272 & $2080 \pm 60$ & $3.6 \times 10^{7}$ \\
\hline${ }^{13} \mathrm{CO} J=6-5^{\mathrm{b}}$ & 645.21 & 453.498 & $<112 \pm 67$ & $8.6 \times 10^{5}$ \\
\hline$\left[\mathrm{C}_{\mathrm{I}}\right]{ }^{3} P_{1}-{ }^{3} P_{0}$ & 480.27 & 609.135 & $1750 \pm 220$ & $1.0 \times 10^{7}$ \\
\hline$[\mathrm{CI}]^{3} P_{2}-{ }^{3} P_{1}$ & 789.95 & 370.415 & $3330 \pm 52$ & $3.1 \times 10^{7}$ \\
\hline$[\mathrm{N} \mathrm{III}]{ }^{3} P_{1}-{ }^{3} P_{0}$ & 1426.1 & 205.300 & $3220 \pm 77$ & $5.5 \times 10^{7}$ \\
\hline $\mathrm{H}_{2} \mathrm{O} 2_{11}-2_{02}$ & 734.22 & 398.643 & $522 \pm 52$ & $4.8 \times 10^{6}$ \\
\hline $\mathrm{H}_{2} \mathrm{O} 2_{02}-1_{11}$ & 964.61 & 303.456 & $798 \pm 75$ & $9.2 \times 10^{6}$ \\
\hline $\mathrm{H}_{2} \mathrm{O} 3_{12}-3_{03}$ & 1071.5 & 273.193 & $548 \pm 60$ & $7.0 \times 10^{6}$ \\
\hline $\mathrm{H}_{2} \mathrm{O} 1_{11}-0_{00}$ & 1086.7 & 269.272 & $234 \pm 52$ & $3.0 \times 10^{6}$ \\
\hline $\mathrm{H}_{2} \mathrm{O} 3_{21}-3_{12}$ & 1135.3 & 257.795 & $606 \pm 60$ & $8.2 \times 10^{6}$ \\
\hline $\mathrm{H}_{2} \mathrm{O} 2_{20}-2_{11}$ & 1199.8 & 243.974 & $394 \pm 60$ & $5.6 \times 10^{6}$ \\
\hline UID line & 1472.7 & 198.689 & $277 \pm 60$ & $4.9 \times 10^{6}$ \\
\hline $\mathrm{H}_{2} v=1-0 S(1)^{\mathrm{c}}$ & & 2.12 & & $3.5 \times 10^{7}$ \\
\hline $\mathrm{H}_{2} v=1-0 S(0)^{\mathrm{c}}$ & & 2.22 & & $1.1 \times 10^{7}$ \\
\hline $\mathrm{H}_{2} v=2-1 S(1)^{\mathrm{c}}$ & & 2.25 & & $4.9 \times 10^{6}$ \\
\hline $\mathrm{H}_{2} v=2-1 S(2)^{\mathrm{c}}$ & & 2.15 & & $2.2 \times 10^{6}$ \\
\hline
\end{tabular}

Notes

a Papadopoulos et al. (2012b).

${ }^{\mathrm{b}}$ Upper limit.

c Tecza et al. (2000), extinction corrected values, luminosities adjusted to adopted distance.

${ }^{\text {d }}$ Relative uncertainties for $L_{\text {line }}$ are the same as for $S_{\text {line. }}$.

of the integration borders). The average is taken as the line flux and the standard deviation of the different realizations is taken as the error, and excludes the relative calibration error of $7 \%$. This is also done for fluxes of lines that are blended. For these, for each iteration, a double Gaussian was fitted and the peaks were used to set the fractional flux for each of the lines. As additional modeling constraints, the table lists fluxes for three lower CO transitions (Papadopoulos et al. 2012b), and luminosities of four $\mathrm{H}_{2}$ transitions (Tecza et al. 2000). From these four $\mathrm{H}_{2}$ lines, Tecza et al. (2000) estimated an unattenuated power of $L_{\mathrm{H}_{2}} \approx 2 \times 10^{9} L_{\odot}$.

\section{ANALYSIS}

We will focus on the excitation of the $\mathrm{CO}$ ladder, and compare $\mathrm{CO}$ excitation conditions to those of $\mathrm{H}_{2}$. A multicomponent Large Velocity Gradient (LVG) analysis of the $\mathrm{CO}$ ladder, considering also constraints from the $\mathrm{HCN}$ and $\left[\mathrm{CI}_{\mathrm{I}}\right]$ lines will be presented in another paper (P. P. Papadopoulos et al., in preparation). Preliminary mass estimates from this study are consistent with those found by Papadopoulos et al. (2012a), Greve et al. (2009), Tacconi et al. (1999), and Engel et al. (2010). Estimates range between $M_{\text {total }}=3 \times 10^{9}$ and $4 \times 10^{10} M_{\odot}$, depending on whether low- and/or high-density tracers are used.

The total luminosity in the ${ }^{12} \mathrm{CO}$ lines listed in Table 1 is $L_{\mathrm{CO}} \approx 5 \times 10^{8} L_{\odot}$. In the absence of $J_{\text {up }}>13$ measurements

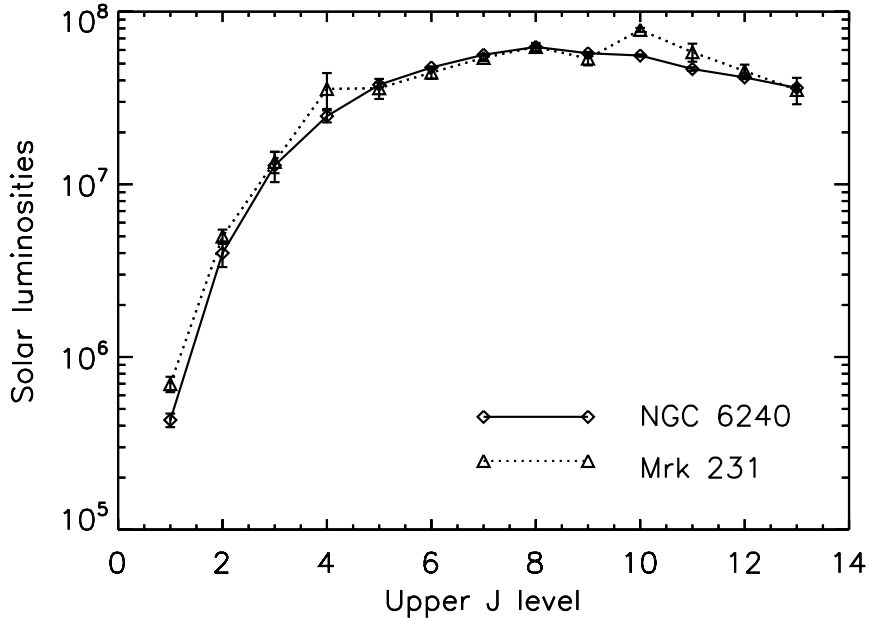

Figure 2. Comparison of the CO ladder of NGC 6240 to those obtained for Mrk 231 (van der Werf et al. 2010). The CO ladder of Mrk 231 is normalized to the CO $J=8-7$ luminosity of NGC 6240 .

this is a lower limit to the total luminosity in the $\mathrm{CO}$ lines. Although the line luminosity of the $\mathrm{CO} J=8-7$ transition is largest, the luminosity in the individual $\mathrm{CO}$ lines is only slowly decreasing for higher rotational quantum numbers, and a significant contribution is expected from transitions with $J_{\text {up }}>13$. The shape of the CO ladder of NGC 6240 is similar to that of Mrk 231 (see Figure 2) and can be fitted by two photondominated region (PDR) models and an X-ray dominated region (XDR) model (see van der Werf et al. 2010). The physical and geometrical properties of NGC 6240 are different from those of Mrk 231. We argue below why the fit used for Mrk 231 is not appropriate for NGC 6240, and why shocks must be responsible for the $\mathrm{CO}$ excitation in NGC 6240.

Absence of $\mathrm{OH}^{+}$and $\mathrm{H}_{2} \mathrm{O}^{+}$. The NGC 6240 spectrum does not show emission line features of the ionic species $\mathrm{OH}^{+}$and $\mathrm{H}_{2} \mathrm{O}^{+}$, observed in Mrk 231 (van der Werf et al. 2010). Large $\mathrm{OH}^{+}$and $\mathrm{H}_{2} \mathrm{O}^{+}$abundances are only sustained in gas clouds with high ionization fractions $\left(x_{e}>10^{-3}\right)$, which are produced by elevated cosmic ray or X-ray fluxes (cf. Meijerink et al. 2011). Their absence hints that the bulk of the gas is not exposed to high ionization rates resulting from active galactic nucleus (AGN) or starburst/supernova activity.

Line-to-continuum ratio. The CO luminosity (up to the $J=$ $13-12$ transition) to infrared (measured between $8-1000 \mu \mathrm{m}$ ) luminosity ratio is $L_{\mathrm{CO}} / L_{\mathrm{IR}}=7 \times 10^{-4}$ in NGC 6240. This is exceptionally high, and approximately an order of magnitude higher than the ratio found for Mrk 231 (van der Werf et al. 2010) and Arp 220 (Rangwala et al. 2011). An exceptionally high line to FIR continuum ratio is also found for the $\mathrm{H}_{2}$ lines of NGC 6240 (van der Werf et al. 1993). Our PDR and XDR models (Meijerink \& Spaans 2005; Meijerink et al. 2007 ) give a maximum $L_{\mathrm{CO}} / L_{\mathrm{IR}}$ ratio of $\sim 10^{-4}$ (assuming $I(\mathrm{FIR})=2.6 \times 10^{-4} G_{0}$ erg s $\mathrm{s}^{-1} \mathrm{~cm}^{-2} \mathrm{sr}^{-1}$; see Kaufman et al. 1999), where the XDR ratios are highest. Most of the absorbed photons in a PDR will heat the dust. An AGN (creating an XDR) generates a UV continuum which contains approximately ten times more energy than the X-ray field, and also heats the dust efficiently. In shocks, on the contrary, the gas is compressed and heated to higher temperatures, while the dust is not affected (except for shock velocities and densities that are orders of magnitude higher). Assuming that shocks are not heating the dust and that all the far-infrared luminosity is reprocessed radiation from the $\mathrm{AGN}$, we obtain a maximum 
AGN contribution of $10 \%-15 \%$. So, a shock-dominated ISM can yield a much larger line-to-continuum ratio than PDRs and XDRs and this is exactly what we see in NGC 6240.

Geometry of NGC 6240. The bulk of the gas mass does not coincide with the two AGNs or with star formation. Engel et al. (2010) relate gas masses, as traced by the $\mathrm{CO} J=2-1$ emission, to the different locations in NGC 6240: $5 \%$ and $25 \%$ to the northern and southern AGNs, and $70 \%$ to the $\mathrm{CO}$ peak in the overlap region, while using apertures of $1^{\prime \prime}$. Our FTS beam $\left(>19^{\prime \prime}\right)$ is larger than the galaxy, and traces the total CO emission. We determined an FWHM of $450 \pm 40 \mathrm{~km} \mathrm{~s}^{-1}$ for the CO $J=13-12$ emission with a gaussian $\times$ sinc profile fit (larger than the instrumental resolution of $245 \mathrm{~km} \mathrm{~s}^{-1}$ at this frequency range). This is similar to the FWHM of the CO $J=2-1$ line at the emission peak between the two nuclei, suggesting that the CO $J=13-12$ emission is also located in this region. The projected distance between these nuclei on the sky is $\sim 750 \mathrm{pc}$, and the true distance between them is estimated at $1.4 \mathrm{kpc}$ (Tecza et al. 2000). These authors assume that the two nuclei are on a circular orbit, of which the position angle and inclination are the same as that of the CO-disk between the two nuclei (Tacconi et al. 1999), and that the velocity difference between the two nuclei is $150 \mathrm{~km} \mathrm{~s}^{-1}$. The AGN X-ray luminosities are not enough to power the $\mathrm{CO}$ excitation of the gas that is residing between the two nuclei, since they are geometrically diluted and absorbed: the luminosities derived by Komossa et al. (2003) give an X-ray flux at $250 \mathrm{pc}$ from the AGN of $<1 \mathrm{erg} \mathrm{cm}^{-2} \mathrm{~s}^{-1}$. Vignati et al. (1999) derive a much larger luminosity by an obscured AGN, with an absorbing screen of $N_{\mathrm{H}} \sim 10^{24} \mathrm{~cm}^{-2}$ which also reduces the X-ray fluxes below $<1 \mathrm{erg} \mathrm{cm}^{-2} \mathrm{~s}^{-1}$. This leaves room for an XDR component located near the AGNs, but even the most optimistic estimate of the X-ray luminosity is not enough to explain the combined $\mathrm{H}_{2}$ and $\mathrm{CO}$ luminosity of $L=2.5 \times 10^{9} L_{\odot}=9.6 \times 10^{42} \mathrm{erg} \mathrm{s}^{-1}$. This would require a strong coupling of the $\mathrm{X}$-rays to the molecular gas.

\subsection{Shock Modeling}

Given our arguments discussed above and the fact that various papers (cf. van der Werf et al. 1993; Tecza et al. 2000) have argued that C-type shocks are exciting the $\mathrm{H}_{2}$ lines in NGC 6240, we analyze the CO ladder with shock models. Two types of shocks are used: a magnetic continuous (C-type) and a non-magnetic jump (J-type) shock model. In both types, rovibrational levels of $\mathrm{H}_{2}$ are excited through hightemperature $\mathrm{H}_{2}-\mathrm{H}_{2}, \mathrm{H}-\mathrm{H}_{2}$, and $\mathrm{He}-\mathrm{H}_{2}$ collisions. As the shock develops, the temperature of the gas becomes such that the excited vibrational states become populated. We use the Flower \& Pineau des Forêts (2003) shock code to model the $\mathrm{H}_{2}$ $v=1-0 S(1) / v=2-1 S(1)$ ratio and the CO ladder. Kristensen et al. (2007) used this model to calculate a grid, spanning hydrogen number densities between $n_{\mathrm{H}}=10^{4}$ and $10^{7} \mathrm{~cm}^{-3}$, velocities between $v=10$ and $50 \mathrm{~km} \mathrm{~s}^{-1}$, and transverse magnetic field densities $b \times n_{\mathrm{H}}^{1 / 2} \mu \mathrm{G}$, with $b=1$ and 5 . The magnetic field density relation implies values between 100 and $3000 \mu \mathrm{G}$ for the adopted densities, which is within the range of values observed for galactic molecular clouds (Crutcher 1999).

Using a rotational diagram and assuming that the $\mathrm{CO}$ lines are optically thin, we find $T_{\text {rot }}=66 \mathrm{~K}$ for the $\mathrm{CO}$ rotational transitions between $\mathrm{CO} J=5-4$ and $J=7-6$. This rotational temperature increases to values $T_{\text {rot }} \sim 150 \mathrm{~K}$ at the highest $\mathrm{CO}$ transitions. We note that for the highest transitions the rotational temperature is a lower limit to the kinetic temperature, since

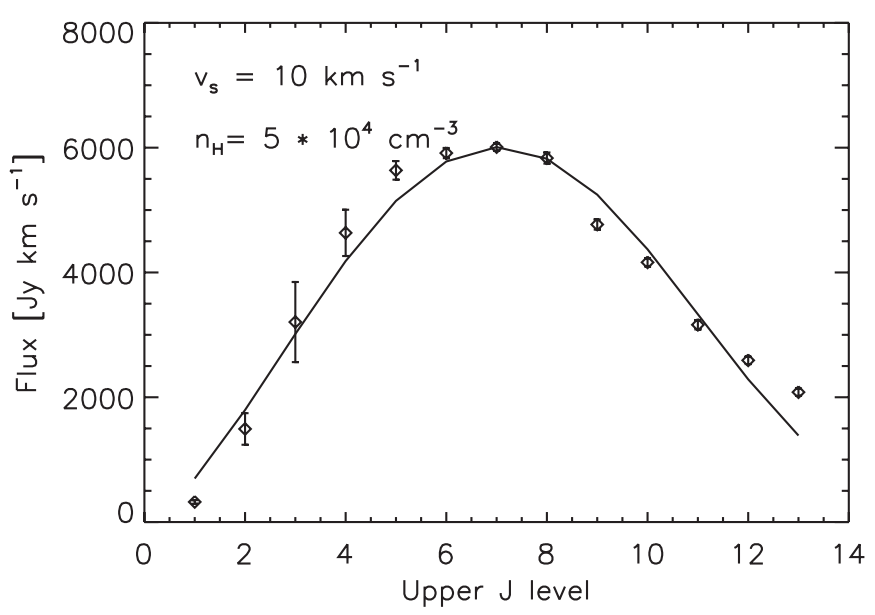

Figure 3. C-shock model (solid line) with density $n_{\mathrm{H}}=5 \times 10^{4} \mathrm{~cm}^{-3}$ and shock velocity $v_{s}=10 \mathrm{~km} \mathrm{~s}^{-1}$ overlaid on the observed CO line fluxes (diamonds) for NGC 6240.

these transitions are slightly sub-thermally excited. Using a $\chi^{2}$ fit and including the errors provided in Table 1 , the bestfitting C-type shock model has a hydrogen number density of $n_{\mathrm{H}}=5 \times 10^{4} \mathrm{~cm}^{-3}$ and velocity of $v=10 \mathrm{~km} \mathrm{~s}^{-1}$ (see Figure 3 ). The density is well constrained, and $\chi^{2}$ values increase by an order of magnitude when going to densities $n=10^{4}$ or $10^{5} \mathrm{~cm}^{-2}$. The uncertainty in the shock velocity is a few $\mathrm{km} \mathrm{s}^{-1}$. Downstream in this particular model, the $\mathrm{CO}$ gas has been compressed by a factor of 7.2 and the post-shock density of the $\mathrm{CO}$ emitting gas is $3.6 \times 10^{5} \mathrm{~cm}^{-3}$.

The $v=1-0 S(1)$ and $v=2-1 S(1) \mathrm{H}_{2}$ lines have upper level energies of $E=6500$ and $12500 \mathrm{~K}$, respectively, and are therefore only excited when the gas temperature $T \gtrsim 1000 \mathrm{~K}$. It turns out that there is a degeneracy between pre-shock density and velocity for reproducing the observed $v=1-0 S(1) / v=$ 2-1 S(1) ratio of $\sim 7$ (Tecza et al. 2000). The lower the pre-shock density, the higher the velocity that is required: the solutions range between hydrogen number density $n_{\mathrm{H}}=5 \times 10^{5} \mathrm{~cm}^{-3}$ and a shock velocity $v_{\mathrm{s}}=47 \mathrm{~km} \mathrm{~s}^{-1}$ to a pre-shock density of $n_{\mathrm{H}}=10^{7} \mathrm{~cm}^{-3}$ combined with a velocity of $v_{\mathrm{s}}=16 \mathrm{~km} \mathrm{~s}^{-1}$.

\section{DISCUSSION AND CONCLUSIONS}

Shock modeling. Combining the model results for the $\mathrm{H}_{2}$ and CO emission, we find that the $\mathrm{H}_{2} v=1-0 S(1)$ to CO $J=10-9$ intensity ratio is of the order of $\sim 100$. In contrast, the observed $\mathrm{H}_{2} / \mathrm{CO}$ line luminosity ratio is approximately $\sim 0.5$. The lowdensity, low-velocity shock model fitting the $\mathrm{CO}$ lines has a low temperature and does not produce $\mathrm{H}_{2}$ emission. From this we conclude that only a very small fraction of the gas mass is currently exposed to very powerful shocks (with either very high densities, $n_{\mathrm{H}}=10^{7} \mathrm{~cm}^{-3}$ and moderate shock velocities $v_{\mathrm{s}}=16 \mathrm{~km} \mathrm{~s}^{-1}$ or moderate densities, $n_{\mathrm{H}}=4 \times 10^{4} \mathrm{~cm}^{-3}$, combined with a high shock velocity $v_{\mathrm{s}} \sim 50 \mathrm{~km} \mathrm{~s}^{-1}$ ). Most of the shocked gas is settling and equilibrating with the ambient ISM, which is in agreement with the fast dissipation timescale derived below.

Dissipation timescales. If we assume that all the gas is colliding at a shock velocity $v_{s}=50 \mathrm{~km} \mathrm{~s}^{-1}$ (which is the highest velocity allowed by the models reproducing the $\mathrm{H}_{2}$ lines), the total amount of energy available (for an adopted gas mass of $M=1.6 \times 10^{10} M_{\odot}$, in the middle of the LVG masses derived by various authors) is $E=0.5 M v_{s}^{2}=4.0 \times 10^{56} \mathrm{erg}$, which is very similar to the value derived by Tacconi et al. 
(1999). Assuming no additional energy input and that CO traces the bulk of the gas mass, this would imply that the $\mathrm{CO}$ shock energy is dissipated away within 6.6 million years, well within the orbital timescale of the two nuclei of 30 million years (Tacconi et al. 1999).

Excitation by $U V / X$-rays versus shocks. The observed $\mathrm{H}_{2}$ $v=1-0 S(1) / v=2-1 S(1)$ ratio falls within the range of ratios that are produced by XDRs (see, e.g., Maloney et al. 1996, Figure 6). Also, the CO ladder of NGC 6240 resembles the one observed for Mrk 231 (van der Werf et al. 2010). However, as mentioned before, NGC 6240 is lacking the bright $\mathrm{OH}^{+}$ and $\mathrm{H}_{2} \mathrm{O}^{+}$lines, which are associated with gas clouds that are exposed to extremely high cosmic ray or X-ray ionization rates (Meijerink et al. 2011), and the available X-ray photons are not sufficient to dominate the chemistry and thermal balance of the bulk of the gas (see Section 3.1). The $\mathrm{H}_{2} \mathrm{O}$ lines are less luminous than in Mrk 231. This implies either lower water abundances or a less efficient mode of excitation. A full analysis is beyond the scope of this Letter. These results and the similarity to the high-J CO line distribution in Mrk 231 suggest that shocks possibly due to the massive molecular outflow (Fischer et al. 2010; Feruglio et al. 2010; Sturm et al. 2011) may also contribute to the CO line emission in Mrk 231. Indeed, based on millimeter interferometric observations of Mrk 231, Cicone et al. (2012) note that in the inner region $(R<0.3 \mathrm{kpc})$, the $\mathrm{CO}(2-1) / \mathrm{CO}(1-0)$ ratio is slightly higher, indicative of a shock contribution.

Observing strategies for ALMA and other submillimeter facilities. Although the observed CO ladders for Mrk 231 and NGC 6240 are practically indistinguishable, we argue that shock excitation and not X-rays are responsible for the excitation of the $\mathrm{CO}$ ladder, based on our knowledge of the geometry of the NGC 6240. Such an analysis will not be possible in the study of the ISM in galaxies at high redshift. At those distances, we are unable to resolve the offset between the two radio nuclei and the location of the $\mathrm{CO}$ emitting gas, and X-ray fluxes cannot be determined. Therefore, we have to rely on dust continuum and line emission at far-infrared and submillimeter wavelengths. Here we have argued that a high line-to-continuum ratio is a key diagnostic for the presence of shocks. Additionally, more detailed modeling of emission by molecules and ions, such as $\mathrm{H}_{2} \mathrm{O}$, $\mathrm{H}_{2} \mathrm{O}^{+}$and $\mathrm{OH}^{+}$, will help in making this distinction, and are thus also highly recommended to include in future ALMA programs.

We acknowledge the constructive comments by the referee Christine Wilson. The following institutes have provided hardware and software elements to the SPIRE project: University of Lethbridge, Canada; NAOC, Beijing, China; CEA Saclay,
CEA Grenoble and LAM in France; IFSI, Rome, and University of Padua, Italy; IAC, Tenerife, Spain; Stockholm Observatory, Sweden; Cardiff University, Imperial College London, UCL-MSSL, STFCRAL, UK ATC Edinburgh, and the University of Sussex in the UK. Funding for SPIRE has been provided by the national agencies of the participating countries and by internal institute funding: CSA in Canada; NAOC in China; CNES, CNRS, and CEA in France; ASI in Italy; MEC in Spain; Stockholm Observatory in Sweden; STFC in the UK; and NASA in the USA. Additional funding support for some instrument activities has been provided by ESA. US authors acknowledge support from the NHSC. Basic research in IR astronomy at NRL is funded by the US ONR.

\section{REFERENCES}

Armus, L., Bernard-Salas, J., Spoon, H. W. W., et al. 2006, ApJ, 640, 204 Cicone, C., Feruglio, C., Maiolino, R., et al. 2012, A\&A, 543, A99 Crutcher, R. M. 1999, ApJ, 520, 706

Depoy, D. L., Becklin, E. E., \& Wynn-Williams, C. G. 1986, ApJ, 307, 116

Elston, R., \& Maloney, P. 1990, ApJ, 357, 91

Engel, H., Davies, R. I., Genzel, R., et al. 2010, A\&A, 524, A56

Feruglio, C., Maiolino, R., Piconcelli, E., et al. 2010, A\&A, 518, L155

Fischer, J., Sturm, E., González-Alfonso, E., et al. 2010, A\&A, 518, L41

Flower, D. R., \& Pineau des Forêts, G. 2003, MNRAS, 343, 390

Greve, T. R., Papadopoulos, P. P., Gao, Y., \& Radford, S. J. E. 2009, ApJ, 692,1432

Griffin, M. J., Abergel, A., Abreu, A., et al. 2010, A\&A, 518, L3

Iono, D., Wilson, C. D., Takakuwa, S., et al. 2007, ApJ, 659, 283

Kaufman, M. J., Wolfire, M. G., Hollenbach, D. J., \& Luhman, M. L. 1999, ApJ, 527,795

Komossa, S., Burwitz, V., Hasinger, G., et al. 2003, ApJL, 582, 15

Kristensen, L. E., Ravkilde, T. L., Field, D., Lemaire, J. L., \& Pineau Des Forêts, G. 2007, A\&A, 469, 561

Maloney, P. R., Hollenbach, D. J., \& Tielens, A. G. G. M. 1996, ApJ, 466, 561

Meijerink, R., \& Spaans, M. 2005, A\&A, 436, 397

Meijerink, R., Spaans, M., \& Israel, F. P. 2007, A\&A, 461, 793

Meijerink, R., Spaans, M., Loenen, A. F., \& van der Werf, P. P. 2011, A\&A, 525, A119

Nakanishi, K., Okumura, S. K., Kohno, K., Kawabe, R., \& Nakagawa, T. 2005, PASJ, 57, 575

Papadopoulos, P. P., van der Werf, P., Xilouris, E., Isaak, K. G., \& Gao, Y. 2012a, ApJ, 751, 10

Papadopoulos, P. P., van der Werf, P., Xilouris, E. M., et al. 2012b, MNRAS, 426, 2601

Rangwala, N., Maloney, P. R., Glenn, J., et al. 2011, ApJ, 743, 94

Rieke, G. H., Cutri, R. M., Black, J. H., et al. 1985, ApJ, 290, 116

Sturm, E., González-Alfonso, E., Veilleux, S., et al. 2011, ApJL, 733, 16

Tacconi, L. J., Genzel, R., Tecza, M., et al. 1999, ApJ, 524, 732

Tecza, M., Genzel, R., Tacconi, L. J., et al. 2000, ApJ, 537, 178

van der Werf, P. P., Genzel, R., Krabbe, A., et al. 1993, ApJ, 405, 522

van der Werf, P. P., Isaak, K. G., Meijerink, R., et al. 2010, A\&A, 518, L42

Vignati, P., Molendi, S., Matt, G., et al. 1999, A\&A, 349, L57

Wilson, C. D., Petitpas, G. R., Iono, D., et al. 2008, ApJS, 178, 189 